\title{
Development and evaluation of an information booklet for adult survivors of cancer in childhood
}

A Blacklay, C Eiser, A Ellis, on behalf of the United Kingdom Children's Cancer Study Group Late Effects Group

\begin{abstract}
Aims-To determine the need for information among survivors of childhood cancer, to assess the acceptability of an information booklet, and to investigate the effectiveness of the booklet in increasing knowledge and influencing health related behaviours.

Subjects-Fifty survivors of childhood cancer (age range 14-32 years) who were consecutive attendees at a long term follow up clinic.

Methods-The booklet was developed for young people aged 14 years and above by the United Kingdom Children's Cancer Study Group Late Effects Group. Included is information about treatment of cancer, general advice about a healthy lifestyle, the rationale for long term follow up, and information about employment and life insurance problems.

Survivors were interviewed at the follow up clinic, offered the booklet, and contacted approximately one week later for a telephone interview. The clinic interview assessed survivors' understanding of their illness and treatment and its impact on their lives, and their preferences for further information. The telephone interview determined survivors' general reaction to the booklet, whether it increased knowledge and influenced health related behaviours.
\end{abstract}

Results-All those interviewed accepted the written information and agreed to a follow up interview. Survivors were enthusiastic about being given more information. Over three quarters learned new information from the booklet. There were no indications that the information was associated with anxiety for any demographic or clinical subgroups. After reading the booklet there was an increased awareness of the risk from sunbathing ( $p<$ 0.05 ), and greater appreciation of the importance of follow up $(p<0.05)$.

Department of

Oncology,

Birmingham

Children's Hospital

A Blacklay

Department of Psychology, University of Exeter, Exeter,

Devon EX4 4GQ

C Eiser

A Ellis

Correspondence to: Dr Eiser.

Accepted 20 October 1997
Recent estimates give an overall five year survival after treatment for malignant disease in excess of $60 \% .^{1}$ It is estimated that there will be 10000 survivors over 16 years of age in the UK by the year 2000. Follow up of survivors of childhood cancer is considered necessary for several reasons.

First, while the likelihood of relapse is small for those who survive more than five years from completion of treatment, ${ }^{2}$ it remains important to monitor any continuing impact of treatment on growth, fertility and other systems, as well as psychological wellbeing. This is necessary to treat any possible sequelae or late effects for the individual, and also to obtain information on which to make decisions about the possible future modification of treatment protocols.

Second, attendance at follow up clinics provides an opportunity to give information to the individual about the possibility of late effects. This approach rests on the assumption that individuals have a right to details about their medical history and that education will be one of the most effective means of influencing health related behaviour.

Survivors need to be aware of the possibility of late effects or a second malignancy resulting directly from the disease or treatment, as well as to understand how their own behaviour might increase or decrease their risk of ill health. Awareness may increase understanding about the reasons for attending follow up clinics, encourage surveillance and health detection behaviours and, in turn, the seeking of help if problems arise. For example, understanding the possible effects of chemotherapy on fertility may allow survivors to come to terms with their situation and limit the risk of emotional disappointment. Appreciation of the possible risks may both discourage health compromising behaviours and encourage health promoting activities. For example, cardiac disease may be a complication of anthracycline chemotherapy ${ }^{3}$ and can be aggravated by work involving heavy weights or sports such as weight lifting. Patients need to understand this rather than risk further compromising their health.

In order to meet these needs for information, an information booklet was developed by members of the United Kingdom Children's Cancer Study Group (UKCCSG) Late Effects Group. The booklet includes general information for all survivors and more specific information relating to the likely consequences of specific aspects of treatment. It is planned that more specific details will be given, depending on individual illness and treatment.
Advances in the treatment of childhood cancer have resulted in a significant increase in the number of survivors entering into adult life. 
The booklet was developed for young people aged 14 years or above, who have remained well for five years after diagnosis. It is planned that the information will be distributed to all survivors through long term follow up clinics. Before national distribution, however, an attempt was made to determine the acceptability of this information to survivors. In this paper, we report survivors' attitudes to the general information only (that is that considered appropriate for all survivors). fold.

The purpose of this paper is therefore three-

(1) Firstly, we aimed to determine whether there is a need for information. This was addressed by investigating the extent to which survivors themselves wanted information, either about their illness or in terms of any implications for future health. Although we have documented the reasons why we think it is important that survivors are informed, we are not aware of whether survivors themselves see such information as necessary. Assessments were made of initial knowledge about the illness, understanding of the reasons for follow up, and awareness of the risk of late effects.

(2) Secondly, we wanted to assess the appropriateness of the proposed booklet. We aimed to evaluate it objectively by assessing its "readability" level, and also subjectively by determining its acceptability to the survivors. Other work suggests that individuals differ in the extent to which they want information about medical treatments. ${ }^{4}$ We therefore attempted to identify subgroups, in terms of clinical or demographic variables, and how they may respond to information of this kind.

(3) While aware that the impact of a brief, focused information booklet is necessarily limited, the ultimate value of such an intervention is in terms of its potential to influence behaviour. In evaluating this booklet, we considered that the criteria for success must include evidence that survivors were informed about (i) the purpose of follow up, (ii) the risks of health compromising behaviours (such as smoking and sunbathing), and (iii) their illness and treatment and any possible impact on the future. Within the scope of this initial survey, it is clearly not possible to evaluate the impact of the booklet in terms of changes in clinic attendance. As in other studies, therefore, we are forced to rely on indirect indicators of success, such as attitude and reported intentions to change behaviour. The third aim, therefore, concerned identifying any impact of the booklet on knowledge, understanding of the rationale for follow up, appreciation of risks, and intention to adopt a healthier lifestyle by reducing risks such as smoking and sunbathing.

\section{Subjects and methods}

SUBJECTS

Participants were 50 young adults ( 25 male, 25 female) who were consecutive attendees at a long term follow up clinic. All those approached agreed to take part. Clinical data were taken from medical records. The mean chronological age was 21.4 years (range 14-32 years), with a mean age at diagnosis of 8.9 years (range 3 months-15 years), and mean time since diagnosis of 12.9 years (range 2-26 years). Thirty survivors had been diagnosed with a solid tumour or Hodgkin's disease, and 20 with leukaemia or non-Hodgkin's lymphoma.

\section{PROCEDURE}

Survivors were initially approached by a doctor (AB), while waiting for a routine check up, and asked if they would be prepared to be interviewed by a psychologist (CE or $\mathrm{AE}$ ) about their illness. There were no refusals. The purpose of the study was explained further and permission requested to tape record the interview. Interviews were conducted in a side ward at the clinic and lasted for approximately 15 minutes.

On completion of the clinic interview survivors were asked if they wished to take an information booklet away with them. They were asked if they were prepared to be contacted within a few days in order to answer some questions about their views of the booklet. If they agreed an arrangement was made to contact them within a one week period. Follow up was by telephone interview.

Both the clinic and follow up interviews were tape recorded and subsequently transcribed.

\section{THE BOOKLET}

From preliminary discussions it was decided that the booklet should be designed for survivors of childhood cancer aged 14 years or above. (At this age survivors could read and appreciate the booklet without help from parents or medical staff.)

The booklet is divided into two main sections. The first includes general information relevant to survivors of all childhood cancers. Information is organised under the following specific sections: types of treatment; reasons for follow up; general advice about health, education, jobs, life insurance, pensions, and having a family; whether cancer could happen again; feelings about yourself, family, and friends. Each section was written by a member of the UKCCSG with special expertise, and the whole coordinated by $\mathrm{AB}$. The second section includes personalised information about the specific cancer experienced as well as details about the treatment received. The evaluation reported in this paper is based on responses to the general information in the first section of the booklet. The readability of the booklet was determined using the Flesch Reading Ease Level using Microsoft Word. ${ }^{5}$

\section{CLINIC INTERVIEW}

The format for both clinic and follow up interviews included both open ended questions and Likert style rating scales. Ratings were made on a series of five point scales with appropriately labelled end points. The clinic interview covered the following issues:

\section{Perceived need for information}

(A) Wish for information-patients were asked to rate whether they thought they knew 
enough about their illness, and also whether they wanted to know any more. In addition, there was an open ended question "Are there any special reasons why you think it might be helpful to have more information about your illness?"

(B) Importance of sections - the separate sections of the booklet (types of treatment; reasons for follow up; etc) were listed and survivors were asked to rate each one in terms of how much they would like information on this topic.

(C) Knowledge of their illness - survivors were asked if they knew the name of the illness and the names of any drugs they had had.

(D) Understanding of the reasons for follow $u p$-reasons for follow up; recall of any explanation for follow up given by parent or doctor.

(E) Impact on the future-perceived impact of the illness on the future.

FOLLOW UP INTERVIEW

Evaluation of the booklet

(A) General evaluation - ratings of the booklet were made in terms of ease of reading and understanding. Survivors were asked how they read the booklet (for example alone, with someone else), what they would do with it now, and whether they would like to see a copy of the final form. Survivors were asked how they thought it should be distributed: by a doctor, by a doctor with an explanation, or made freely available in the clinic. They were also asked if they were upset in any way by the contents of the booklet.

(B) Evaluation of the booklet sections-ratings were made for each of the sections as to whether information was included which survivors wanted to know.

(C) New information gained - this was an open ended question where survivors were asked to report any new information acquired.

\section{Changes from clinic interview to follow up interview}

The following were included in both interviews, in order to determine changes in awareness that might be attributable to reading the booklet.

(A) Perceived importance of attending follow $u p$ - ratings were made on a five point scale from 1 "not at all important" to 5 "very important".

(B) Risks in relation to friends-survivors rated their own risks of smoking, sunbathing, and strenuous activities in comparison with their friends. Again ratings were made on five point scales from 1 "a lot less risk than my friends" to five "a lot more risk than my friends"

(C) Intentions to change behaviour-beliefs about what could be done to stay well in the future and any intentions to make changes in behaviour.

\section{STATISTICAL ANALYSIS}

Content analyses were conducted on the open ended questions. All data were entered into a mainframe computer and the data analysed using SPSS-X.

Relationships between continuous variables were assessed using Pearson's correlation coefficients, and the relationships between categorical variables were analysed using $\chi^{2}$ statistics. Changes in ratings from clinic to follow up interview were assessed using $t$ tests.

\section{Results}

READABILITY

The booklet was found to have a Flesch Reading Ease of 59.2, which suggests that it is appropriate for those over 14 years or in year 9 or above.

\section{CLINIC INTERVIEW}

Perceived need for information

(A) Wish for information- $72 \%$ of survivors felt they did not know enough about their illness and $62 \%$ wanted to know more. The reasons given for wanting information were because medication was still needed for example for growth or hormonal problems; because they had to explain to others, especially employers, about their limited skills or abilities; or because they were embarrassed by their appearance. Sixteen per cent knew they could not have children and were seeking help or advice. Those who did not want any more information felt their illness was in the past and had no implications for their future.

(B) Importance of sections - in terms of the separate booklet sections, fewer survivors were interested in information about education, jobs, and life insurance (41\%) or feelings about yourself, family, and friends (56\%). Interest in the remaining sections was high (reasons for follow up, $67 \%$; general health advice, $67 \%$; having a family, $83 \%$; and second malignancy, $94 \%)$.

(C) Knowledge of the illness- $56 \%$ of the group could name their cancer, $34 \%$ could only give a general name (for example cancer, tumour), and $10 \%$ could not give any information about their illness. Sixty six per cent did not know the names of any drugs they had been given, $12 \%$ reported general class names (steroids), and the remainder could name specific drugs.

(D) Understanding of the reasons for follow $u p$ - the most common reason given for attending follow up was that it was a precautionary measure to check their general progress $(44 \%)$. Thirty per cent thought it was to check for second malignancy, $18 \%$ that it was for research purpose, and only $8 \%$ that it was to check for treatment late effects. Forty eight per cent of survivors could not remember that anyone had ever discussed the reason for follow up with them, $44 \%$ had been told by a doctor, and $8 \%$ by a parent.

(E) Impact on the future- $50 \%$ of patients thought the illness would have a negative effect on their future. They were concerned about limited job opportunities, infertility, and physical late effects. Forty per cent felt that their illness was in the past and did not anticipate any complications or side effects. The remaining $10 \%$ thought the illness would have a positive 
impact on their future citing feelings of greater maturity, an increased ability to cope, a positive attitude to life, and greater health awareness.

On completion of the interview, all survivors were enthusiastic to take a copy of the booklet and agreed to the follow up interview.

FOLLOW UP INTERVIEW

Forty seven follow up interviews were conducted. Three survivors could not be contacted and were not at home at the times arranged. (These three had all rated, during the clinic interview, that they did not perceive any need for further information.)

\section{Evaluation of the booklet}

(A) General evaluation - all those who were interviewed were very positive about the value of the booklet. Eighty seven per cent found it easy to read and understand. Eighty seven per cent read the booklet alone. When asked what they would now do with it, $85 \%$ said that they would keep it, $13 \%$ said they would keep it and show it to someone else, and $2 \%(\mathrm{n}=1)$ said they would throw it away. All but one wished to see the final version.

Respondents thought that the most appropriate method of distribution was that a doctor should discuss the booklet with the survivor during a clinic consultation. Eight per cent thought it should be given by a doctor but felt it did not need discussion, and $22 \%$ thought it should be made freely available in clinics for people to take or not as they wished.

Sixty seven per cent said they were not upset or distressed in any way by the booklet. The remainder did not like being reminded about the treatment, or felt upset for children who were now having to undergo such experiences. However, they all accepted that the information was important, and did not regret having been given it.

(B) Evaluation of the booklet sections - the separate sections of the booklet were rated positively, with almost $70 \%$ reporting that the information was appropriate and answered their questions. The exception was the section concerning recurrence, where just $55 \%$ felt their questions were answered.

\section{Criticisms of the booklet}

There were no major criticisms of the booklet, though $38 \%$ would like further information, especially with regard to fertility and their own personal vulnerability.

\section{Changes from clinic interview to follow up interview}

(A) Impact of the booklet on knowledge-81\% reported that they learned at least one new piece of information from the booklet (reasons for follow up, $28 \%$; general advice about health, $42 \%$; education, life insurance, $8 \%$; fertility issues, $16 \%$; second cancers, $6 \%$ ).

(B) The importance of follow up - there was an increased awareness of the importance of follow up after reading the booklet; $65 \%$ (ratings of 4 or 5) thought it important at clinic interview compared with $95 \%$ (ratings of 4 or
5) at follow up interview. A paired $t$ test on the mean scores indicated that this was a significant increase $(t=2.13, \mathrm{df}=38, \mathrm{p}<0.05)$.

(C) Risks with respect to friends - a paired $t$ test on the mean scores indicated there was a non-significant increase in the number of survivors reporting that smoking was particularly bad for them ( $70 \%$ (ratings of 4 or 5$)$ initially thought they were at greater risk from smoking than their peers compared with $77 \%$ (ratings of 4 or 5) at follow up). There was a similar non-significant shift in awareness of risks associated with strenuous activities (from 12 to $28 \%$ giving ratings of 4 or 5 ).

However, a paired $t$ test on the means scores indicated there was a significant increase in awareness of the dangers of sunbathing ( $t=$ 2.45 , df $=40, \mathrm{p}<0.05)$. Twenty two per cent (ratings of 4 or 5) thought they were at greater risk that than peers from sunbathing before hand compared with $59 \%$ (ratings of 4 or 5 ) at follow up.

Four survivors ( $8 \%$ ) were smokers. All realised that they were at greater risk from smoking then their friends and two stated that, because of the booklet, they would try to give up.

(D) Intentions to change behaviour- $40 \%$ intended to make changes in their behaviour in order to keep healthy. These included paying greater attention to diet, weight control, more regular exercise, greater care when sunbathing, more regular visits to the dentist, and breast self examinations.

In addition to the general health promotion issues, other practical advice was obtained from the booklet. This included greater insight into how to obtain insurance, intentions to consult a doctor when considering starting a family, and help in formulating questions that they wished to ask at their next follow up check.

Reaction to the booklet as a function of

demographics and initial opinions

There were no significant associations between age, sex, age at diagnosis, time since diagnosis, and whether survivors were upset by the booklet, or whether they still wanted more information having read it. However, survivors with a diagnosis of a solid tumour learned more than those with leukaemia $\left(\chi^{2}=5.7, \mathrm{p}<0.05\right)$.

\section{Discussion}

Our data suggest that knowledge in this group of survivors was generally low both about past treatment and the possible implications for the future. One tenth of survivors could not name their illness in the simplest of terms (cancer or tumour). Surprisingly few studies have assessed knowledge in children with cancer, though there have been occasional reports drawing similar conclusions. ${ }^{6}$

Awareness of the possibility of late effects was also limited. Only half considered that their illness might have a negative effect on their future, and only one tenth thought one of the reasons for attending follow up clinics was to check for late effects. Few survivors recalled that a doctor had discussed the reasons for attending follow up clinics with them. This 
finding highlights the special difficulties of working with survivors of childhood cancer, in that it may be unclear when is the most appropriate time to raise questions about late effects and need for regular follow up.

It may be that these children were not previously given much information about their illness or that they have forgotten or chosen to ignore any suggestions that they may experience problems in the future. It seems that some patients (both adults and children) cope with long term illness by minimising the severity of their condition. ${ }^{4}$ In our efforts to inform patients, we must remain sensitive to the needs of those who choose to adopt this approach to managing their lives. Information may be helpful for some, but not all, patients, and for this reason individual rights to not know need to be respected.

However, our data suggest that the majority of these survivors of childhood cancer welcome the availability of information both about their past medical history as well as about any future consequences of the illness. While some are simply curious about their pasts, others need the information in order to deal with ongoing consequences of the illness. Information is especially important for those who require further treatment, especially for growth or hormone problems, for those with visible sequelae and for those seeking employment. It is important that survivors' very real needs for information are considered sympathetically and met in long term follow up clinics.

Although this group of survivors were aware of their increased risk from smoking compared with their friends, they did not appear to appreciate any additional risks which may be associated with sunbathing or strenuous activities. These findings are consistent with previous work, ${ }^{6}$ which suggests that some areas of general health promotion may be over emphasised in clinics at the expense of others.

We were unable to identify individual differences in response to the booklet, at least in terms of the clinical and demographic variables investigated. The exception is diagnostic group. As might be expected, given that they form a more heterogeneous group, those with solid tumours learned more compared with those with leukaemia. We were unable to identify any subgroups who were distressed by the information provided. The implication so far is that there do not appear to be certain groups of survivors for whom information of this kind is not appropriate.

It is important to use some discretion when distributing the booklets. Since many were not aware of the names of specific drugs they had been given, it would be possible for them to interpret some sections of the booklet inappropriately. Some anxiety may be caused if survivors read the general information, without professional support, since they may be concerned unnecessarily about risks that do not in fact affect them.

The effectiveness of this brief, focused intervention is particularly reflected in the findings that survivors subsequently viewed attendance at follow up clinic as more important. In addition, some $40 \%$ expressed some intention to alter their behaviour as a result of reading the booklet. While we recognise that good intentions are rarely enough to change behaviour, it is encouraging that such a relatively high percentage at least expressed interest. It is also important to note that this was a minimal intervention; survivors were given the booklet in clinic with only a brief explanation. In practice, we believe it is important that the contents of the booklet are discussed during clinic visits, and the key messages reinforced on subsequent appointments. This preliminary evaluation was conducted in an established long term follow up setting, which means that the survivors included may be relatively well informed about possible late effects. Further work is necessary to determine the impact of the information among those who are more naive about possible consequences of treatment.

The success of the booklet was apparent in that (i) well over three quarters of the survivors learned something new, (ii) there was a significant change in awareness of risks from sunbathing, and (iii) there was increased awareness of the importance of follow up. There were no changes in perceived risks associated with smoking (although the incidence of smoking in this group was low, and the majority were already aware of the associated risks) or from strenuous activities.

Although some survivors wanted more information than was provided, this was most frequently with respect to their own treatment. The intention has always been to include personal information about treatment when the booklet becomes available for wider distribution. This will clarify specific treatments experienced and how individuals may expect to be affected. As a result of this evaluation, some revisions have been made in order to reduce the reading age. It is now planned that the booklet will be distributed through long term follow up clinics. In addition, future work will aim to develop age appropriate information for younger survivors.

C Eiser and A Ellis are funded by the Cancer Research Campaign, CP 1019/0101. We would like to thank Serono Laboratories for funding the publication of the booklet.

Members of the UKCCSG Late Effects Group: M Stevens, MEM Jenney (chair), MM Hawkins, G Levitt, M Sokal, M MEM Jenney (chair), MM Hawkins, G Levitt, M Sokal, M
Brada, J Kingston, H Wallace, D Williams, G Kissen, R Skinner, Brada, J Kingst
and H Davies.

1 Boring CC, Squires TS, Tong T, Montgomery S. Cancer statistics. CA Cancer $\mathcal{F}$ Clin 1994;44:7.

2 Robertson CM, Hawkins MM, Kingston JE. Causes of late death after childhood cancer and implications for cure. BMF 1994;309:162-6.

3 Hawkins MM, Stevens MCG. The long term survivors. $\mathrm{Br}$ Med Bull 1996;52:898-923.

4 Miller SM. Monitoring versus blunting styles of coping with cancer influence the information patients want and need about their disease; implications for cancer screening and management. Cancer 1995;76:167-77.

5 Flesch RF. A new readability yardstick. f Appl Psychol 1948; 32:221-33.

6 Eiser C, Levitt G, Leiper A, Havermans T, Donovan C. Clinic audit for long-term survivors of childhood cancer. Arch Dis Child 1996;75:405-9. 